\title{
Analysis of Indoor Rowing Motion using Wearable Inertial Sensors
}

\author{
Stephan Bosch \\ Department of Computer \\ Science \\ University of Twente \\ The Netherlands \\ s.bosch@utwente.nl \\ Lennart Buit \\ I.j.buit@student.utwente.nl
}

\author{
Muhammad Shoaib \\ Department of Computer \\ Science \\ University of Twente \\ The Netherlands \\ m.shoaib@utwente.nl \\ Nirvana Meratnia \\ Department of Computer \\ Science \\ University of Twente \\ The Netherlands \\ n.meratnia@utwente.nl
}

\author{
Stephen Geerlings \\ s.a.geerlings@student.utwente.nl
}

\begin{abstract}
In this exploratory work the motion of rowers is analyzed while rowing on a rowing machine. This is performed using inertial sensors that measure the orientation at several positions on the body. Using these measurements, this work provides a preliminary analysis of the differences between experienced and novice rowers, or between a good and a bad technique. The analysis shows that the measured postural angles show no clear trend that would set apart experienced and novice rowers or a bad and a good technique. However, there are clear differences in absolute postural angle's consistency and timing consistency of strokes between novice and experienced rowers. We also applied a machine learning technique to the data to find the similarities between different rowers and an experienced reference rower. The results can be used to compare the quality of the rowing technique with respect to a reference. In this paper, we present our initial results as well as the challenges that need to be further explored.
\end{abstract}

\section{Keywords}

Rowing, inertial motion capture, body sensor network, machine learning

\section{INTRODUCTION}

Rowing is a sport in which performance is determined by technique and strength. While building strength is relatively simple, novice rowers need a great amount of guidance from a coach to learn the proper technique and optimize their performance. Normally, most of the technique is learned on dry land using a rowing machine (ergometer). A coach can then clearly see what aspects of the technique need improvement. However, guidance from a coach is not always readily available, for example in a regular gymnasium. Volunteer coaches are a scarce resource for many rowing associations. Therefore, it would be nice to have a system with which a rower can get real-time feedback on his performance without a coach present or a coach can later on analyze the rowing motion, for example, for consistency in postural angles and timing.

To solve these issues, rowers could be monitored with a sensor system. With the sensor measurements, the system can provide information about a rower's motion during rowing activity. Using this information, the coach can better evaluate the crew's performance. When there is no coach, the system could provide that information directly to the athletes, possibly accompanied with suggestions on how performance could be improved. Alternatively, the data could be recorded for evaluation by a coach at a later time.

The performance of a rower can be assessed in a number of ways. For rowing exercise on the water, the movement of the boat and the oars can be monitored using sensors [5]. For indoor rowing, the rowing machine can be instrumented to measure various parameters of the rower's activity, such as power output, exerted force and the position of the slide and handle bar [3]. Alternatively, the posture of the rower can be measured using cameras or on-body sensors [17] .

There are proprietary systems to measure the behavior and performance of rowers in a boat. These systems are very costly and are mostly designed for professional athletes [19]. They consist of many different sensors that generate information mostly about power output and stroke rate. However, the sensors often cannot detect errors in rowing posture or timing. Rowing technique is largely composed of posture and timing and this work aims to explore these aspects further.

The goal of this research is to measure and analyze rowing motion and to develop a method to give feedback for the 
rowers or coaches. Information on the posture of a rower can provide insight in flaws in the rower's technique that directly influence performance [17]. Moreover, bad posture can lead to injury $[6,24]$. In this work, we compare the motion characteristics of experienced rowers to novice rowers, to determine what constitutes a correct rowing technique. We compare the posture of the rowers and the timing of their strokes. Analysis of the measured movement could be used to advise a rower what needs to be improved. Timing and posture information will be essential to advise the rower properly. This system can assist the rowers and trainers if it is easy to use and if it gives real-time feedback.

Our goal is to eventually build a system that can assess posture correctness during training on the water. However, for simplicity, we limit our experiments outlined in this paper to the rowing machine. We use on-body inertial motion sensors, because the alternative camera-based motion tracking systems are not suitable for measurement on the water and they would be prohibitively expensive for most amateur rowing associations. The disadvantage of using motion sensors is that this technique provides less accurate and less reliable measurements in most cases.

Our contributions are as follows:

- We provide a preliminary exploration of the differences in posture and timing between experienced and novice rowers. A similar approach can be used for differentiating between a good and an inferior rowing technique.

- We apply machine learning to assess the similarity between the rower's movements.

- We make our data set and videos publicly available for future research in this domain [22].

This work is explicitly limited to determining the distinction between experienced and novice rowers. This is based on the assumption that experienced rowers will have better technique than novice rowers. This is not necessarily true however, since an experienced rower can have a bad day for instance. We aim to extend this work for determining what can be improved in the rower's technique. Using expert supervision, data on good rowing technique can be collected for a large set of athletes, making this work suitable for determining whether rowers use the correct technique, irrespective of whether they are experienced or not. The system could also be personalized by using an expert to determine a good rowing technique for one particular rower. This way, the performance variation of one rower can be measured relative to the personal reference obtained with expert guidance. This could be very useful for subsequent unsupervised training.

The rest of the paper is organized as follows. In Section 2, we briefly describe related work. Some background regarding rowing technique is provided in Section 3. Subsequently, we describe our experimental setup in Section 4 and we describe the results in Section 5. Finally, we describe our conclusions and future work in Section 6.

\section{RELATED WORK}

Wearable sensors are extensively used in sports research, especially for analyzing the performance of elite athletes $[2,11$, 16]. For rowing, most research focuses either on the athlete's force or power output $[2-5,8]$ and the optimal movement of the oars $[1,5,12,18,19]$ and the boat $[18,19]$. The posture of the athlete received less attention so far, while it does provide deeper insight in flaws in the rower's technique that directly influence performance. Moreover, bad posture can lead to injury $[6,24]$.

Hawkins et al. [14], use electrogoniometers to capture the joint angles during indoor rowing experiments similar to ours. Since these mechanical devices need to be strapped along the joints, these can constrain the rower's movements. A similar setup by Page et al. [21] uses a mechanical system involving potentiometers to measure the angles. Using an on-body network of wearable sensors to track the rower's posture can be less obtrusive, and more importantly it can be used on the water. A usability study by Franke et al. [9] demonstrates that wireless body sensors similar to ours do not constrain the rower in any way.

King et al. [17] perform experiments very similar to ours using a rowing machine and three wireless inertial sensor nodes. Although King et al. demonstrate examples of what bad technique looks like, they mainly investigate the feasibility of the measurement setup and they do not compare measurements between rowers. Tanaka et al. [24] perform posture comparisons between experienced and novice rowers, but they don't use on-body sensors and they focus entirely on preventing injury.

\section{BACKGROUND}

A rowing stroke has roughly two fundamental reference points: the catch is the moment at which the oar blade enters the water and the finish (also known as the extraction, release, or tapping down) is the moment at which the blade is finally removed from the water. At the catch position, the rower is fully bent towards the back of the boat. While the oar is in the water, the rower applies force to the oar, thereby pushing the boat forward. This is called the drive phase of the stroke. Once the oar is lifted above the water, the rower moves from the finish position back to the catch position, which is called the recovery phase of the stroke.

Much of the rowing technique revolves around timing and posture. For example, the rower needs to lean forward by the appropriate amount, needs to pull back on the oar at the correct time, the rower should keep his back straight, should pull backwards with his upper body at the correct time, etc. Therefore, when we compare experienced and novice rowers, we expect to see differences in the measured posture and the timing of the various steps in the rowing stroke.

\section{EXPERIMENTS}

All experiments are performed on dry land with indoor rowing machines (ergometers). Figure 1 shows an overview of the experimental setup. The rower subjects are equipped with three inertial sensor modules located at one lower leg, the lower back and the upper back. These locations are chosen based on expert knowledge. The placement of the 


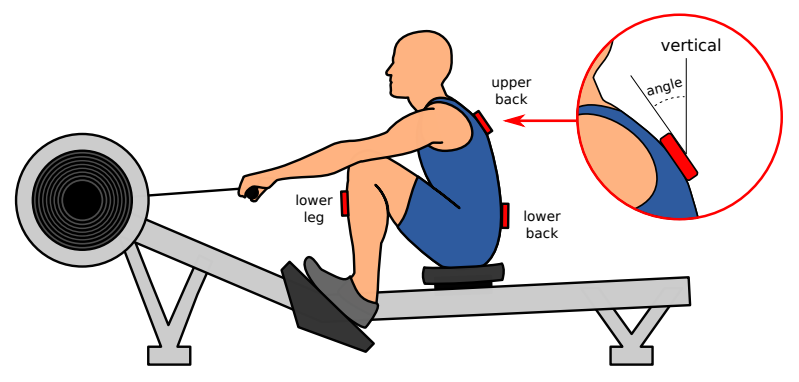

Figure 1: An overview of the experiment with the locations of the sensor modules.

sensors allows measurement of the main posture angles during the rower's motion; i.e., the angle of the lower leg and the angle of the torso. The two sensors on the back provide a measurement of the extent at which the back is curved.

The sensor modules use accelerometer and gyroscope sensors to measure the module's own acceleration and rotational velocity respectively. Using these measurements, we determine the orientation of the sensors during the experiment using the algorithm by Madgwick et al. [20]. This yields the orientation of the sensors represented in quaternions.

Using these orientation measurements, we determine the angle of each sensor relative to the vertical, as shown in Figure 1. We only evaluate the motion in a two-dimensional fashion, meaning that we assume that there is no significant sideways motion at the sensor positions [24]. With this assumption, we can use the shortest angle between the sensor orientation and the vertical. To verify the angle measurements, we made video recordings [22] of the experiments from a perspective similar to Figure 1 .

We use the ProMove-3D [15] system from Inertia Technology for this work. A ProMove-3D sensor node features a 3-D accelerometer, a 3-D gyroscope and a 3-D digital compass. The accelerometer has a measurement range of up to $\pm 6 \mathrm{~g}$ and the gyroscope has a measurement range of up to $\pm 2000^{\circ} \mathrm{s}^{-1}$. The nodes form a network using a $2.4 \mathrm{GHz}$ radio. The wireless network is used to transmit sensor measurements to a computer for recording and to synchronize measurements from multiple sensor nodes within $10 \mu \mathrm{s}$.

The sample frequency for all sensors was set to $200 \mathrm{~Hz}$. The wireless transmission of the data caused a sample loss of up to $2 \%$, which is compensated for by means of linear interpolation.

For this work we evaluate the motion characteristics of both experienced rowers (rowing for three years or more) and novice rowers (rowing for a few months at most). Since this is only a preliminary study, we perform experiments with a limited set of only seven subjects, of which three are experienced and four are novice rowers. The characteristics of our subjects is shown in Table 1.

To determine whether the rowing speed is a factor, the rowers all perform successive experiments with 20 and 30 strokes

\begin{tabular}{cccc}
\hline \hline & Height $\mathbf{( c m )}$ & Weight $\mathbf{( k g )}$ & Age (years) \\
\hline \hline Experienced 1 & 196 & 71 & 23 \\
Experienced 2 & 183 & 81 & 25 \\
Experienced 3 & 191 & 93 & 21 \\
Novice 1 & 180 & 70 & 24 \\
Novice 2 & 184 & 74 & 19 \\
Novice 3 & 180 & 72 & 20 \\
Novice 4 & 185 & 67 & 20 \\
\hline
\end{tabular}

Table 1: Experiment subject characteristics

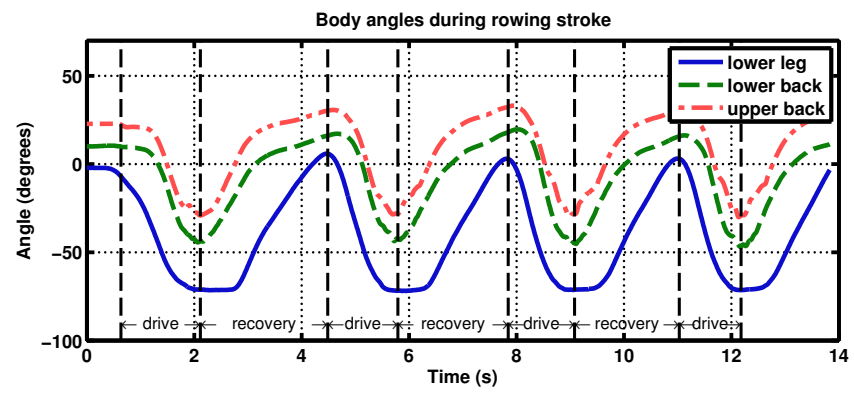

Figure 2: Example of the sensor angles during rowing at 20 strokes per minute.

per minute. The rowers use feedback from the rowing machine to maintain the requested stroke rate. Each experiment involves approximately 20 strokes, all of which are used in our evaluation (whole session). Each experiment at a specific stroke rate is performed only once by each individual rower.

\section{RESULTS}

For part of this analysis, we choose one experienced rower as a reference, which we label as Exp. 1. This is the rower with the most average physique, which means that he matches best with all the other experienced and novice rowers. The other experienced rowers are labeled with successive numbers and the novice rowers are labeled as e.g. Nov. 1. Rower Exp. 2 was female and rower Exp. 3 was male, but with a stronger physique than our reference. Measurements at 30 strokes per minute for rower Exp. 3 failed and were not used for this analysis.

Figure 2 shows an example of the angle measurements from our reference rower performing the first four strokes at 20 strokes per minute. The figure is annotated with the drive and recovery phases of the stroke cycle. These phases are determined automatically using a simple heuristic algorithm that bases the phase transitions on peaks in the lower leg and upper back angle signals. The drive phase starts (at the catch position) when the lower leg starts to move backwards (angle decreasing) and ends (at the finish position) when the upper back starts to move forwards (angle increasing). We use these annotations to compare the stroke phases separately and align our comparisons between rowers.

Our goal is to find measurable posture characteristics that distinguish an experienced rower from a novice rower, or rather characteristics that distinguish between a good and a bad technique. We evaluate several possibilities: we compare the absolute angles and its consistency, and the timing 
consistency of rowing strokes. These characteristics were selected based on expert knowledge on what constitutes a good rowing technique, which largely revolves around good posture and timing. Finally, we employ machine learning techniques to obtain a generic measure of similarity.

\subsection{Absolute Angles}

The most obvious way to compare rowing postures is using the absolute angles measured during the experiment. Therefore, with the help of literature and expert knowledge, we devised a simple quantitative model in which the reference angles for a certain positions in the stroke phase are defined. In an effort to determine these reference angles, we evaluated the angles from our experienced rowers.

Figure 3 shows the angle differences between our reference rower and each of the other novice and experienced rowers at the upper back sensor at a velocity of 20 strokes per minute. The results are compensated for differences in the length of the strokes by normalization; the $\mathrm{x}$-axis shows the phase of the full stroke rather than an absolute time. The angle differences are only shown at the stroke phase positions of the reference angles of our quantitative model.

The results show large differences in the angles, even amongst the experienced rowers. This is a clear indication that the absolute angle has very little value for comparing rowing posture. The results for the leg and lower back positions are very similar. We speculate the measured angles do not only depend on the rower's technique, but that there are also significant dependencies on the rower's physique. The rower's length and strength are likely important factors. However, these are not the only factors, since rowers with similar physique (such as Exp. 1 and Exp. 2) still show significant differences. For a stroke rate of 30 strokes per minute, we observe very similar results as for the experiment at 20 strokes per minute.

Figure 4 shows the standard deviations in the leg, lower back and upper back angles at a velocity of 20 strokes per minute. These statistics show that on average the experienced rowers have lower standard deviation values than inexperienced rowers. This standard deviation can be an indication of the angle consistency of the rowers. We expect a high standard deviation for rowers with an inconsistent, bad technique. For example, Nov. 3 has a high standard deviation for all angles, which is a likely indicator for an inconsistent technique.

\subsection{Stroke Timing Consistency}

Table 2 shows the stroke timings for each rower at 20 strokes per minute. The ideal stroke at that speed lasts 3 seconds. The table shows that the experienced rowers are able to match this timing more closely. More interestingly, the standard deviation of the full stroke time is smaller for the experienced rowers, meaning that their strokes are more consistent. The results show that on average about $40 \%$ of a stroke is spent in the drive phase, which is consistent with the stroke proportion statistics reported by Dawson et al. [7]. Judging by the standard deviation, the fraction of time spent in the drive phase is also more consistent for the experienced rowers. The results for the recovery phase are not shown in

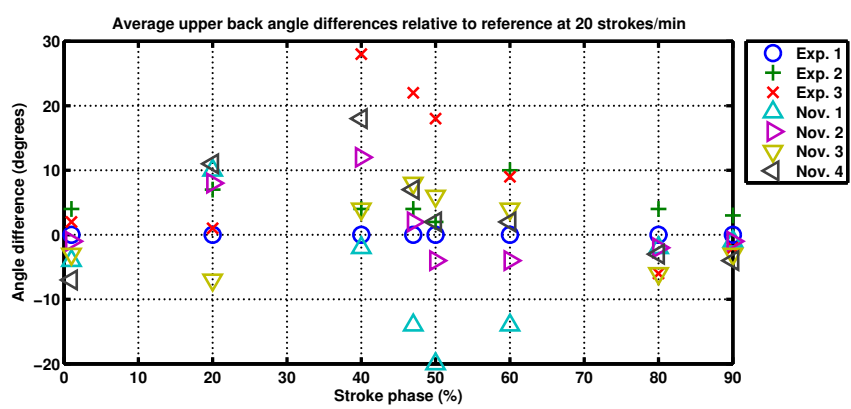

Figure 3: Average upper back angle differences at 20 strokes per minute.

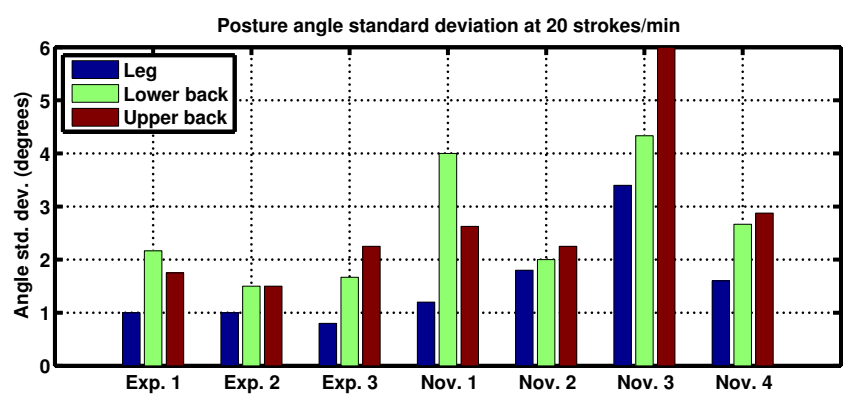

Figure 4: Posture angle standard deviation at 20 strokes/min.

\begin{tabular}{ccccc}
\hline \hline & \multicolumn{2}{c}{ Full Stroke } & \multicolumn{2}{c}{ Drive } \\
& time(s) & std.dev.(s) & time frac.(\%) & std.dev.(\%) \\
\hline \hline Experienced 1 & 3.06 & 0.04 & 40.00 & 2.00 \\
Experienced 2 & 2.90 & 0.04 & 37.00 & 1.00 \\
Experienced 3 & 2.95 & 0.07 & 36.00 & 1.00 \\
Novice 1 & 2.40 & 0.14 & 44.00 & 2.00 \\
Novice 2 & 2.87 & 0.17 & 38.00 & 3.00 \\
Novice 3 & 2.73 & 0.16 & 35.00 & 5.00 \\
Novice 4 & 2.92 & 0.11 & 44.00 & 2.00 \\
\hline \hline
\end{tabular}

Table 2: Stroke timing consistency for 20 strokes per minute

Table 2, because this is the only other phase and therefore the statistics are identical.

Table 3 shows the stroke timings for each rower 30 strokes per minute. The results show that the stroke timing consistency of experienced rowers is not noticeably affected by the stroke rate. In contrast, statistics for both the full stroke and the fraction spent in the drive phase indicate that novice rowers can maintain more consistent stroke timing at higher stroke rates. Figure 5 presents an overview of the standard deviations for the full stroke at each stroke rate for each of our subjects, which shows this stroke rate dependence more clearly. At the highest evaluated stroke rate, the standard deviation of the full stroke is for most novice rowers at the same level as the experienced rowers. Still, at that stroke rate the fraction of a full stroke spent in the drive phase still shows higher standard deviation for the novice rowers.

While a higher stroke rate may improve stroke timing con- 


\begin{tabular}{ccccc}
\hline \hline & \multicolumn{2}{c}{ Full Stroke } & \multicolumn{2}{c}{ Drive } \\
& time (s) & std.dev.(s) & time frac.(\%) & std.dev.(\%) \\
\hline \hline Experienced 1 & 1.91 & 0.06 & 45.00 & 1.00 \\
Experienced 2 & 1.97 & 0.06 & 44.00 & 1.00 \\
Experienced 3 & N/A & N/A & N/A & N/A \\
Novice 1 & 1.83 & 0.05 & 47.00 & 1.00 \\
Novice 2 & 1.88 & 0.13 & 44.00 & 2.00 \\
Novice 3 & 1.95 & 0.06 & 44.00 & 2.00 \\
Novice 4 & 2.03 & 0.06 & 43.00 & 2.00 \\
\hline \hline
\end{tabular}

Table 3: Stroke timing consistency for 30 strokes per minute

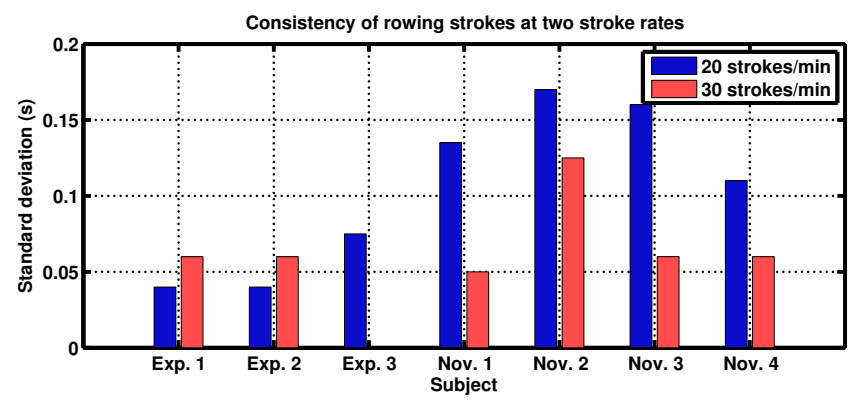

Figure 5: Overview of stroke time consistency.

sistency for novice rowers, the results in Section 5.3 indicate that the overall technique of the novice rowers becomes less similar to the experienced rowers at higher stroke rates. We speculate that it becomes more difficult to maintain correct technique at higher stroke rates.

\subsection{Machine Learning}

It is difficult to determine all aspects of the motion that distinguish experienced rowers from novice ones or a bad rowing technique from a good one. Therefore, we employ machine learning techniques to obtain a generic measure of similarity of the motion of subjects in our experiments relative to the motion of an experienced reference rower.

Obviously, we expect and assume that the motion of our experienced rowers will be more similar to the reference experienced rower than the novice rowers. However, if the novice rower is doing the rowing technique in the right way, then it can have a better similarity to the reference rower as well. Moreover, we also assume that the reference rower is doing the technique in the right way, which is not a very strong assumption. However, this assumption was necessary to keep the scope of this preliminary study limited. In future data collection experiments, the reference rower will be supervised by an expert where we can be sure that the reference technique is a proper one.

These machine learning results will not be useful in determining what exactly is different or wrong. However, this assessment will provide an indication of how much a rower is deviating from the reference. Such indication can be helpful for the rowers to ask for a supervised session to evaluate their technique. This approach can be very helpful in personalized training. For example, a rower can be supervised by an expert to perform the rowing technique once and use that data as a reference point, thereby training a personalized classifier. Such a personalized classifier can detect deviations from the verified technique for that specific rower, when the expert or trainer is not around. Moreover, it can help him by giving a generic feedback about such deviations. This personalized approach is more practical than the generic ones. However, we leave this for the next study as a future work.

We employ machine learning to recognize the two main phases of a rowing stroke: drive and recovery. We train our classifiers using these two stroke phases. The classifiers are trained using our reference rower, which is one of the experienced rowers, and subsequently applied to each of the other rowers as a test data. The recognition performance is used as the measure for similarity. We have used one rower as a reference because of our limited data set. In future, we are planning to cluster experienced rowers for training a classifier.

We used WEKA (version: 3.7) [13] as a machine learning tool for analysis. For our initial analysis, we selected Knearest neighbor for finding similarities between different rowers. It uses Euclidean distance to find similarity between training data and test data. We use this classifier in its default mode in order to make our work easily reproducible, except $\mathrm{K}$ is chosen as 3 . This specific number is chosen, because an odd number is important for breaking the possible tie in majority voting. Also, higher values of $\mathrm{K}$ help in avoiding the effects of noise [23].

We segmented the angle measurements into windows of 75 samples $(0.375 \mathrm{~s})$ with no overlap. This way, at least two window segments are fully located in either the drive or recovery phase of each stroke. Using a sliding window approach, we extracted the following features from the segmented angle measurements: the mean and the mean angular velocity. The mean angular velocity is calculated by taking the mean of the differences between each two consecutive points within each segment. Our two classes were of different sizes. Therefore, we used the classbalancer filter [25] on our training data in the preprocessing phase. This filter assigns the same weights to all classes, thereby avoiding bias towards the majority class by various classifiers [25].

For performance evaluation, we use accuracy, and Matthews Correlation Coefficient (MCC). MCC is considered a balanced measure for the recognition performance, because it considers all aspects of a confusion matrix [10]. These are defined as follows as per WEKA documentation:

- The True Positive rate (TPR) or accuracy is the proportion of examples which were classified as class $\mathrm{x}$, among all examples which truly have class $\mathrm{x}$, i.e. what part of the class was captured correctly.

- The MCC takes into account all aspects of a confusion matrix, such as true positive (TP), true negative $(\mathrm{TN})$, false positive $(\mathrm{FP})$, and false negative $(\mathrm{FN})$ and is calculated as follows:

$$
M C C=\frac{(T P * T N-F P * F N)}{\sqrt{(T P+F P)(T P+F N)(T N+F P)(T N+F N)}}
$$


The results for the KNN classifier at 20 and 30 strokes per minute are respectively shown in Figure 6 and Figure 7 . It is important to note that these results are the average of the drive and recovery phases. These results show how much the recognition performance of all rowers is lower than that of the reference rower Exp. 1. The reference rower is one of the three experienced rowers.

As expected, the similarity to our reference rower is consistently better for our experienced rowers than for our novice rowers with a few exceptions. In some cases, the inexperienced rowers have almost the same or better similarity to the reference rower than that of the experienced rowers. A possible explanation for this could be that the inexperienced rower is doing the technique properly at that specific time. We repeated this analysis for the other two experienced rowers (Exp. 2 and Exp. 3), by taking each of them as a reference point and then evaluating the rest of the rowers with respect to them. We see similar trends.

That is one of the limitations of this study, where we assumed that inexperienced rowers will not perform the rowing technique in an optimal way compared to the experienced ones. That assumption can be wrong, so this should be explored further. One option could be to have an expert present at the data collection phase who can supervise the process and identify who is doing the technique in a proper way.

Beside this analysis, we analyzed these results in relationship with the heights of the rowers to see if it plays an important role. However, we do not see any clear connection between them.

It can also be seen that in some cases the performance drop for these rowers compared to the reference rower or compared to each other, is small. Based on expert knowledge, such small differences can be ignored by applying a threshold in comparing these performances. Because a good rowing technique does allow for different angle values within a specific range. Defining this specific range is the job of a rowing expert and it can be easily integrated with such intelligent systems.

Though this work should be further explored, we observe that different performance measures can be combined to differentiate between an experienced and inexperienced rower or a good and bad technique. As we discussed in Section 5.2, stroke timing consistency becomes better at higher stroke rates, whereas the similarity with the reference becomes worse at higher stroke rates for novice rowers. Therefore, we can combine these two factors for distinguishing experienced from novice rowers or distinguishing a bad technique from a good one, because at least one of these will likely differ.

\section{CONCLUSION}

Our preliminary experiments show that absolute posture angles provide no direct means of distinguishing experienced and novice rowers. For the absolute posture angles, the measurements vary wildly, even amongst experienced rowers. However, the standard deviation of the absolute postural angles could be used to assess the inconsistency of the

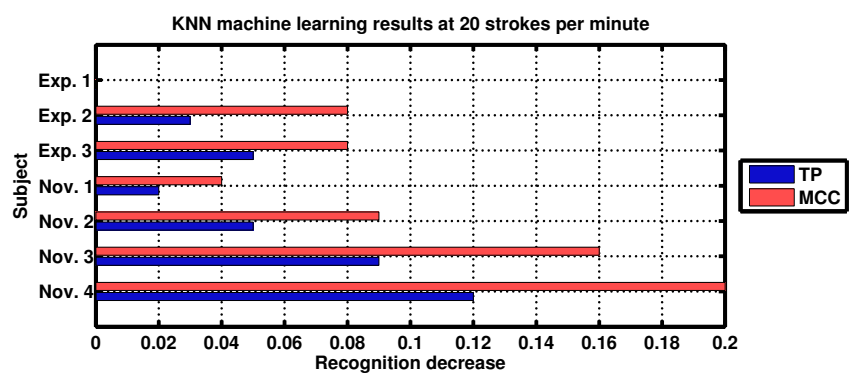

Figure 6: Machine learning results for the KNN classifier at 20 strokes per minute.

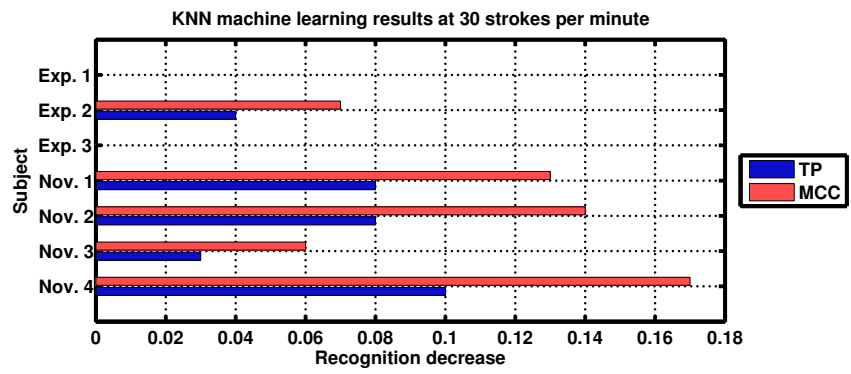

Figure 7: Machine learning results for the KNN classifier at 30 strokes per minute.

rower's technique.

In contrast, the timing consistency of the rowing strokes does show a clear difference between experienced and novice rowers. The standard deviation of the full stroke time and the fraction of the stroke spent in the drive phase is low and consistent across varying stroke rates for the experienced rowers. Novice rowers show higher standard deviation. Interestingly, the consistency of novice rowers improves with higher stroke rates.

Machine learning techniques can distinguish between experienced and novice rowers, because the recognition performance of the latter is lower compared to our reference. Unlike the stroke timing consistency, at higher stroke rates, the recognition performance using machine learning of the novice rowers becomes low compared to the experienced reference rower. Therefore, a combination of these two metrics can be used to identify a deviant rowing technique.

An important problem with using machine learning for this analysis is that it is not possible to tell exactly what an inexperienced rower is doing different from the 'good' technique. This requires further exploration. Moreover, we assumed that our inexperienced rowers would consistently perform worse than our experienced rowers. This does not account for some natural talent, which means that inexperienced rowers could perform equally well in some cases. For future experiments, we should have an expert present that can judge the technique during the experiment to make a clear assessment. 
In future work, the number of subjects needs to be larger than seven and experiments need to be repeated several times. With only a few subjects each performing only a few experiments, the results can be influenced by unapparent coincidental factors, such as the participants suffering from fatigue, illness, or stress. Also, our experiments did not explicitly explore the effects of gender differences on rowing posture: most of our subjects were male, with only one exception.

\section{Acknowledgment}

This work is supported by Dutch National Program COMMIT in the context of SWELL project.

\section{REFERENCES}

[1] R. P. Andrews, A. P. Garcia, B. R. Dryer, S. F. Bonney, S. Badjou, and D. E. Dow. Rowing Training System for On-the-water Rehabilitation and Sport. In Proceedings of the 8th International Conference on Body Area Networks, BodyNets '13, pages 351-354, ICST, Brussels, Belgium, Belgium, 2013. ICST (Institute for Computer Sciences, Social-Informatics and Telecommunications Engineering).

[2] A. Baca and P. Kornfeind. Rapid Feedback Systems for Elite Sports Training. IEEE Pervasive Computing, 5(4):70-76, Oct. 2006.

[3] M. Bassetti, F. Braghin, F. Cheli, and S. Maldifassi. Instrumented Rowing Machine for Optimized Training. In R. Allemang, J. D. Clerck, C. Niezrecki, and J. R. Blough, editors, Topics in Modal Analysis II, Volume 6, Conference Proceedings of the Society for Experimental Mechanics Series, pages 499-506. Springer New York, Jan. 2012.

[4] A. Baudouin, D. Hawkins, and S. Seiler. A biomechanical review of factors affecting rowing performance. British Journal of Sports Medicine, 36(6):396-402, Dec. 2002.

[5] S. Bettinelli, A. Placido, L. Susmel, and R. Tovo. An Integrated Data Acquisition System for on-Water Measurement of Performance in Rowing. Strain, 46(5):493-509, Oct. 2010.

[6] A. M. J. Bull and A. H. McGregor. Measuring spinal motion in rowers: the use of an electromagnetic device. Clinical Biomechanics, 15(10):772-776, Dec. 2000.

[7] R. G. Dawson, R. J. Lockwood, J. D. Wilson, and G. Freeman. The Rowing Cycle: Sources of Variance and Invariance in Ergometer and On-the-Water Performance. Journal of Motor Behavior, 30(1):33-43, Mar. 1998.

[8] S. Fothergill. Examining the effect of real-time visual feedback on the quality of rowing technique. Procedia Engineering, 2(2):3083-3088, June 2010.

[9] T. Franke, C. Pieringer, and P. Lukowicz. How Should a Wearable Rowing Trainer Look Like? A User Study. In 2011 15th Annual International Symposium on Wearable Computers (ISWC), pages 15-18, June 2011.

[10] A. Gensler and B. Sick. Novel criteria to measure performance of time series segmentation techniques. In Proceedings of the 16th LWA Workshops: KDML, IR and FGWM, September 8-10 2014.

[11] D. Gouwanda and S. M. N. A. Senanayake. Emerging Trends of Body-Mounted Sensors in Sports and
Human Gait Analysis. In D. N. A. A. Osman, D. F. Ibrahim, D. W. A. B. W. Abas, H. S. A. Rahman, and D. H.-N. Ting, editors, 4th Kuala Lumpur International Conference on Biomedical Engineering 2008, number 21 in IFMBE Proceedings, pages 715-718. Springer Berlin Heidelberg, Jan. 2008.

[12] F. Gravenhorst, A. Muaremi, F. Kottmann, G. Troster, R. Sigrist, N. Gerig, and C. Draper. Strap and row: Rowing technique analysis based on inertial measurement units implemented in mobile phones. In 2014 IEEE Ninth International Conference on Intelligent Sensors, Sensor Networks and Information Processing (ISSNIP), pages 1-6, Apr. 2014.

[13] M. Hall, E. Frank, G. Holmes, B. Pfahringer, P. Reutemann, and I. H. Witten. The weka data mining software: an update. $A C M$ SIGKDD explorations newsletter, 11(1):10-18, 2009.

[14] D. Hawkins. A new instrumentation system for training rowers. Journal of Biomechanics, 33(2):241-245, Feb. 2000.

[15] Inertia Technology. ProMove 3D - 3D motion tracking.

[16] D. A. James. The Application of Inertial Sensors in Elite Sports Monitoring. In E. F. Moritz and S. Haake, editors, The Engineering of Sport 6, pages 289-294. Springer New York, Jan. 2006.

[17] R. King, D. McIlwraith, B. Lo, J. Pansiot, A. McGregor, and G.-Z. Yang. Body Sensor Networks for Monitoring Rowing Technique. In Sixth International Workshop on Wearable and Implantable Body Sensor Networks, 2009. BSN 2009, pages 251-255, June 2009.

[18] J. Llosa, I. Vilajosana, X. Vilajosana, and J. Marques. Design of a Motion Detector to Monitor Rowing Performance Based on Wireless Sensor Networks. In International Conference on Intelligent Networking and Collaborative Systems, 2009. INCOS '09, pages 397-400, Nov. 2009

[19] J. Llosa, I. Vilajosana, X. Vilajosana, N. Navarro, E. SuriÃśach, and J. M. MarquÃís. REMOTE, a Wireless Sensor Network Based System to Monitor Rowing Performance. Sensors, 9(9):7069-7082, Sept. 2009.

[20] S. Madgwick, A. Harrison, and R. Vaidyanathan. Estimation of IMU and MARG orientation using a gradient descent algorithm. In 2011 IEEE International Conference on Rehabilitation Robotics (ICORR), pages 1-7, June 2011.

[21] P. N. Page and D. A. Hawkins. A real-time biomechanical feedback system for training rowers. Sports Engineering, 6(2):67-79, June 2003.

[22] Pervasive Systems Research Data Sets. http://ps.ewi.utwente.nl/Datasets.php.

[23] Y. Song, J. Huang, D. Zhou, H. Zha, and C. L. Giles. Iknn: Informative k-nearest neighbor pattern classification. In Knowledge Discovery in Databases: PKDD 2007, pages 248-264. Springer, 2007.

[24] C. Tanaka, M. R. Ide, and R. T. Moreno. Quantitative analysis of head and trunk posture in rowers during ergonometric training. Salusvita, 26(1):53-64, 2007.

[25] Weka documentation for class balancer:. http://weka.sourceforge.net/doc.dev/weka/ filters/supervised/instance/ClassBalancer.html. 